\title{
A Reliable Approach for Sustainable Telehealth Partnerships
}

Bryan T. Arkwright

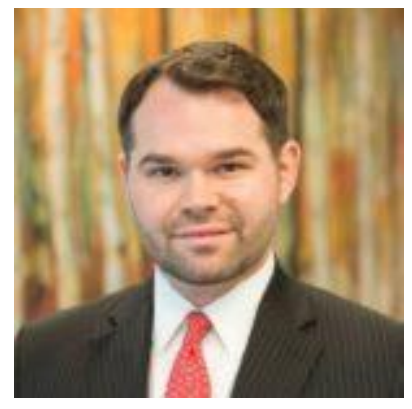

Telehealth is transforming the healthcare industry and creating a paradigm shift in how patients in the U.S. and abroad access high-quality healthcare. A key to that success is creating a sustainable and trusted relationship between the stakeholders who deliver, facilitate, and receive telehealth services. The last five years have proved telehealth to be a catalyst for a plethora of new and emerging models of how different healthcare stakeholder groups interact and innovate with one another.

A review of those involved in high performing telehealth models reveals the following key stakeholders at varying levels:

- Patients

- Health Systems/Hospitals

- Health Clinics

- Long-term Care Facilities

- Physicians and Care Providers

- Technology Vendors

- Service Vendors

- Government

- Payers

- Grantors and Grantees

- Start-ups/Entrepreneurs

- Venture Capital 
Assessing the telehealth market and industry shows numerous models of engagement and involvement from each of these stakeholders. No matter which stakeholder group you align with most, understanding and validating shared telehealth objectives, goals, and incentives are key to creating a long-term sustainable and trusted relationship.

As an active advocate and practitioner of telehealth services I regularly use a simple approach for telehealth stakeholders to assist in everything from early brainstorming through contract negotiations to ongoing operational optimization, which can be used across multiple stakeholder relationships. The telehealth stakeholder incentives approach is illustrated as a visual Venn Diagram in Figure 1 and expanded upon here.

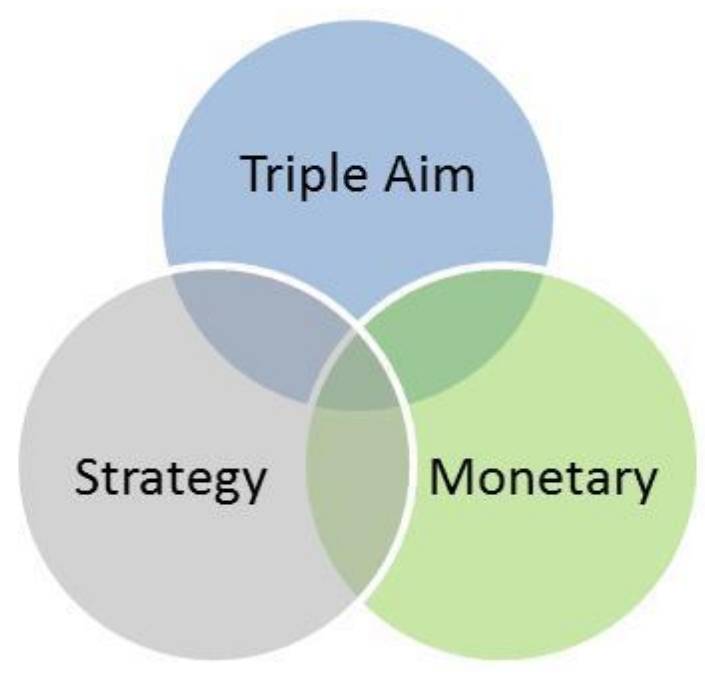

Figure 1. Telehealth stakeholder incentives approach

\section{Triple Aim}

Developed by the Institute for Healthcare Improvement $(\mathrm{IHI}),{ }^{1}$ the IHI Triple Aim is a framework that describes an approach to optimize healthcare system performance and deliver high-quality patient care. The focus of the Triple Aim is to improve population health, reduce per capita cost, and improve the experience of care. These three focus areas create an excellent starting point when considering a telehealth relationship of 
any kind. Delivering the Triple Aim is possible with telehealth when all stakeholders have a similar mission and vision that commit to achieving the Triple Aim together.

\section{Strategy}

A clear strategy that benefits the stakeholders involved in a mutually beneficial way is critical. ${ }^{2} \mathrm{~A}$ telehealth strategy will differ widely by stakeholder and is based on numerous factors. Stakeholders should seek to share and understand each potential stakeholder's strategy. Having an overlapping or complementing strategy for telehealth stakeholders guides the relationship towards an engaged, winning and sustainable relationship.

\section{Monetary}

The telehealth market continues to grow in size year-over-year in investment capital, consumption, benefits, and products/offerings/solutions. ${ }^{3}$ Being clear about the investment, return, and ongoing costs associated with any telehealth relationship from all stakeholders is an important part of the discussion. Having open dialogue about direct and indirect benefits is essential, as telehealth innovation is synonymous with emerging business models. Understanding the return on investment and monetary aspects for each stakeholder is essential to long-term sustainability.

\section{The Sweet Spot and Conclusion}

This approach to understand telehealth stakeholder incentives can be a simple way to assess and discuss new and mature telehealth opportunities. Whichever stakeholder group you align with, understanding how you interact with other stakeholders using the Venn Diagram is critical to building trust and establishing a sustainable telehealth partnership.

Bryan Arkwright is Senior Consultant at Schumacher Clinical Partners; Telehealth/Telemedicine Strategy and Operations. He is also a member of the Telehealth and Medicine Today Editorial Board. 


\section{References}

1. Institute for Healthcare Improvement. The IHI Triple Aim. URL: http://www.ihi.org/engage/initiatives/tripleaim/pages/default.aspx. Accessed 8/14/16.

2. Hecht A, Shin A, Matousek A. Telehealth and health IT policy: Considerations for stakeholders. ML Strategies Alert. URL: https://www.mintz.com/newsletter/2014/Advisories/4220-0914-NATMLS/index.html. Accessed 11/3/16.

3. Anonymous. Telemedicine Statistics Show Big Growth Potential. Healthcare Trends Institute. 2016. URL: http://www.wexhealthinc.com/healthcare-trendsinstitute/telemedicine-statistics-show-big-growth-potential/. Accessed 11/3/16.

Tags:

Arkwright, care providers, government, grantors and grantees, health clinics, health systems/hospitals, how does telehealth doctor patient relationship, long-term care facilities, monetary, patients, payers, physicians, service vendors, startups/entrepreneurs, strategy, technology vendors, telehealth, telemedicine, telemedicine process improvement, telemedicine program implementation, Triple Aim, venture capital, what is telemedicine and how does it work 